\title{
ON REAL HYPERSURFACES OF A COMPLEX SPACE FORM. WITH $\eta$-PARALLEL RICCI TENSOR
}

\author{
By
}

Young Jin SuH*

\section{Introduction.}

Let $M_{n}(c)$ denote an $n$-dimensional complex space form with constant holomorphic sectional curvature $c$. It is well known that a complete and simply connected complex space form consists of a complex projective space $\boldsymbol{C P}^{n}$, a complex Euclidean space $\boldsymbol{C}^{n}$ or a complex hyperbolic space $\boldsymbol{C} \boldsymbol{H}^{n}$, according as $c>0, c=0$ or $c<0$. In this paper we consider a real hypersurface $M$ of $\boldsymbol{C P} \boldsymbol{P}^{n}$ or $\boldsymbol{C H}^{n}$.

The study of real hypersurfaces of $\boldsymbol{C} \boldsymbol{P}^{n}$ was initiated by Takagi [10], who proved that all homogeneous hypersurfaces of $\boldsymbol{C P}^{n}$ could be divided into six types which are said to be of type $A_{1}, A_{2}, B, C, D$ and $E$. Moreover, he showed that if a real hypersurface $M$ of $\boldsymbol{C P}^{n}$ has two or three distinct constant principal curvatures, then $M$ is locally congruent to one of the homogeneous ones of type $A_{1}, A_{2}$ and $B([11])$. Recently, to give another charac terization of homogeneous hypersurfaces of type $A_{1}, A_{2}$ and $B$ in $\boldsymbol{C P}^{n}$ Kimura and Maeda [6] introduced the notion of an $\eta$-parallel second fundamental form, which was defined by $g\left(\left(\nabla_{X} A\right) Y, Z\right)=0$ for any vector fields $X, Y$ and $Z$ orthogonal to the structure vector field $\xi$, where $A$ means the second fundamental form of $M$ in $\boldsymbol{C P} \boldsymbol{P}^{n}$, and $g$ and $\nabla$ denote the induced Riemannian metric and the induced Riemannian connection, respectively.

On the other hand, real hypersurfaces of $\boldsymbol{C H}^{n}$ have also been investigated by many authors (Berndt [1], Montiel [8], Montiel and Romero [9]).

Using some results about focal sets, Berndt [1] proved the following.

THEOREM A. Let $M$ be a connected real hypersurface of $C H^{n}(n \geqq 2)$. Then $M$ has constant principal curvatures and $\xi$ is principal if and only if $M$ is locally congruent to one of the following.

\section{$\left(A_{0}\right)$ a horosphere in $\mathrm{CH}^{n}$.}

Received September 16, 1988. Revised January 24, 1989.

*) Partially supported by TGRC-KOSEF. 
$\left(A_{1}\right)$ a geodesic hypersphere or a tube over a complex hyperbolic hyperplane $\mathrm{CH}^{n-1}$.

$\left(A_{2}\right)$ a tube over a totally geodesic submanifold $C H^{k}$ for $k=1, \cdots, n-2$.

(B) a tube over a totally real hyperbolic space $R H^{n}$.

It is necessary to remark that real hypersurfaces of type $A_{0}$ or $A_{1}$ appearing in Theorem $\mathrm{A}$, are totally $\eta$-umblical hypersurfaces with two distinct constant principal curvatures. In the paper of Montiel [7] the real hypersurface of type $A_{0}$ in Theorem $\mathrm{A}$ is said to be self-tube.

In $\S 3$ we also consider the $\eta$-parallel second fundamental form in $\boldsymbol{C H}^{n}$ and give a further characterization of type $A_{0}, A_{1}, A_{2}$, and $B$ in $\boldsymbol{C} \boldsymbol{H}^{n}$. Now we introduce the notion of an $\eta$-parallel Ricci-tensor of $M$ in $M_{n}(c), c \neq 0$, which is defined by $g\left(\left(\nabla_{X} S\right) Y, Z\right)=0$ for any $X, Y$, and $Z$ orthogonal to $\xi$, where $S$ is the Ricci-tensor of $M$ in $M_{n}(c), c \neq 0$. It is easily seen that if the second fundamental form is $\eta$-parallel, then so is the Ricci-tensor, under the condition such that $\xi$ is principal. Thus the purpose of this paper is to investigate this converse problem. By using the classification theorem due to Takagi [10] and Kimura and Maeda [6], we get the following.

THeOREM B. Let $M$ be a real hypersurface of $\boldsymbol{C P}^{n}$. Then the Ricci-tensor of $M$ is $\eta$-parallel and $\xi$ is principal if and only if $M$ is locally congruent to one of homogeneous real hypersurfaces of type $A_{1}, A_{2}$ and $B$.

By applying the Theorem A we can also prove the following.

THeOREM C. Let $M$ be a real hypersurface of $\boldsymbol{C H}^{n}(n \geqq 2)$. Then the Riccitensor of $M$ is $\eta$-parallel and $\xi$ is principal if and only if $M$ is locally congruent to one of type $A_{0}, A_{1}, A_{2}$ and $B$.

\section{§1. Preliminaries.}

Let $M$ be a real hypersurface of a complex $n$-dimensional complex space form $M_{n}(c)$, and let $C$ be its unit normal vector field. Since $M_{n}(c)$ admits an almost complex structure, let us denote by $F$ its almost complex structure. For any tangent vector field $X$ and normal vector field $C$ on $M$, the transformations of $X$ and $C$ under $F$ can be given by

$$
F X=\phi X+\eta(X) C, \quad F C=-\xi,
$$

where $\phi$ defines a skew-symmetric transformation on the tangent bundle $T M$ of 
$M$, while $\eta$ and $\xi$ denote a 1 -form and a vector field on a neighborhood of $x$ in $M$, respectively. In which it is seen that $g(\xi, X)=\eta(X)$, where $g$ denotes the induced Riemannian metric on $M$. By the properties of the almost complex structure $F$, they satisfy the following

$$
\phi^{2}=-I+\eta \otimes \xi, \quad \phi \xi=0, \quad \eta(\phi X)=0, \quad \eta(\xi)=1,
$$

where $I$ denotes the identity transformation. The set of tensors $(\phi, \xi, \eta, g)$ is called an almost contact structure on $M$.

Furthermore, the covariant derivatives of the structure tensors are given by

$$
\left(\nabla_{X} \phi\right) Y=\eta(Y) A X-g(A X, Y) \xi, \quad \nabla_{X} \xi=\phi A X,
$$

where $\nabla$ is the induced Riemannian connection of $g$ and $A$ denotes the shape operator with respect to $C$ on $M$.

Since the ambient space $M_{n}(c)$ is of constant holomorphic sectional curvature $c$, the equation of Gauss and Codazzi are respectively given as follows:

$$
\begin{aligned}
& R(X, Y) Z \\
& =c\{g(Y, Z) X-g(X, Z) Y+g(\phi Y, Z) \phi X-g(\phi X, Z) \phi Y-2 g(\phi X, Y) \phi Z\} / 4 \\
& \quad+g(A Y, Z) A X-g(A X, Z) A Y, \\
& \quad\left(\nabla_{X} A\right) Y-\left(\nabla_{Y} A\right) X=c\{\eta(X) \phi Y-\eta(Y) \phi X-2 g(\phi X, Y) \xi\} / 4,
\end{aligned}
$$

where $R$ denotes the Riemannian curvature tensor of $M$ and $\nabla_{X} A$ denotes the covariant derivative of the shape operator $A$ with respect to $X$.

The Ricci-tensor $S^{\prime}$ of $M$ is the tensor of type $(0,2)$ given by $S^{\prime}(X, Y)=$ $\operatorname{tr}\{Z \rightarrow R(Z, X) Y\}$. Also it may be regarded as the tensor of type $(1,1)$ and denoted by $S: T M \rightarrow T M$; it satisfies $S^{\prime}(X, Y)=g(S X, Y)$. From (1.3) we see that the Ricci tensor $S$ of $M$ is given by

$$
S=c\{(2 n+1) I-3 \eta \otimes \xi\} / 4+h A-A^{2},
$$

where we have put $h=\operatorname{Tr} A$. The covariant derivative of (1.5) are given as follows

$$
\left(\nabla_{X} S\right) Y=\frac{c}{4}\left\{-3\left(\nabla_{X} \eta\right)(Y) \xi-3 \eta(Y) \nabla_{X} \xi\right\}+(X h) A Y+h\left(\nabla_{X} A\right) Y-\left(\nabla_{X} A^{2}\right) Y .
$$

The Ricci-tensor on the real hypersurface of $M_{n}(c), c \neq 0$, is said to be $\eta$ parallel if it satisfies $g\left(\left(\nabla_{X} S\right) Y, Z\right)=0$ for any tangent vector fields $X, Y$, and $Z$ in $\xi^{\perp}$. In the sequel, assume that the hypersurface $M$ is with $\eta$-parallel Ricci-tensor. Thus for any $X, Y$, and $Z$ in $\xi^{\perp},(1.6)$ gives

$$
g\left(\left(\nabla_{X} S\right) Y, Z\right)=(X h) g(A Y, Z)+h g\left(\left(\nabla_{X} A\right) Y, Z\right)-g\left(\left(\nabla_{X} A^{2}\right) Y, Z\right)=0 .
$$


It follows from (1.7) that if $\xi$ is principal and if the second fundamental form is $\eta$-parallel, then the Ricci-tensor is $\eta$-parallel.

\section{§2. Certain lemmas.}

Let $M$ be a real hypersurface of a complex space form $M_{n}(c), c \neq 0$. The shape operator $A$ of $M$ can be considered as a symmetric $(2 n-1,2 n-1)$-matrix. Now we suppose that the structure vector $\xi$ is a principal curvature vector of $A$, that is, $A \xi=\alpha \xi$, where $\alpha$ is the principal curvature corresponding to $\xi$.

Then the covariant derivative gives

$$
\left(\nabla_{X} A\right) \xi=(X \alpha) \xi+\alpha \phi A X-A \phi A X,
$$

where we have used the second formular of (1.2). Thus it follows that

$$
g\left(\left(\nabla_{X} A\right) Y, \xi\right)=(X \alpha) \eta(Y)+\alpha g(Y, \phi A X)-g(Y, A \phi A X),
$$

for any tangent vector fields $X$, and $Y$ on $M$. By using the equation of Codazzi to (2.1) and using the fact $X \boldsymbol{\alpha}=(\xi \alpha) \eta(X)$, we have

$$
2 A \phi A X-c \phi X / 2=\alpha(\phi A+A \phi) X .
$$

We now introduce the following fact without proof.

LEMMA 2.1. ([3]) Let $M$ be a real hypersurface of $M_{n}(c), c \neq 0$. If $\xi$ is $a$ principal curvature vector of $A$, then its principal curvature $\alpha$ is locally constant.

REMARK. Maeda [7] proved that $\alpha$ is constant for the real hypersurface of $\boldsymbol{C P}$.

Since $\boldsymbol{C} \boldsymbol{P}^{n}$ has constant holomorphic sectional curvature $c=4,(2.2)$ gives the following.

LeMMA 2.2. ([7]) Let $M$ be a real hypersurface of $C P^{n}$. Assume that $\xi$ is a principal curvature vector and the corresponding principal curvature is $\alpha$. If $A X=\lambda X$ for any $X$ in $\xi^{\perp}$, then $A \phi X=((\alpha \lambda+2) /(2 \lambda-\alpha)) \phi X$.

\section{§ 3. Real hypersurfaces of $C H^{n}$ with $\eta$-parallel second fundamental form.}

It is well known that the complex hyperbolic space $\boldsymbol{C H}^{n}$ admits the Bergmann metric normalized so that the constant holomorphic sectional curvature $c$ is -4 .

Thus (2.2) gives the following equation for the real hypersurface of $\boldsymbol{C H}^{n}$ whose structure vector field $\xi$ is principal. 


$$
2 A \phi A X+2 \phi X=\alpha(\phi A+A \phi) X
$$

for any tangent vector field $X$ in $M$. It follows that if $A X=\lambda X$ for any $X$ in $\xi^{\perp}$, then

$$
(2 \lambda-\alpha) A \phi X=(\alpha \lambda-2) \phi X .
$$

Now we need the following lemmas which will be used in the later.

LEMMA 3.1. (Montiel and Romero [9]) Let $M$ be a real hypersurface of $C H^{n}$. Then

$$
A \phi=\phi A \text { holds on } M \text { if and only if } M \text { is of type } A_{0}, A_{1} \text { or } A_{2} \text {. }
$$

LeMMA 3.2. Let $M$ be a real hypersurface of $\mathrm{CH}^{n}$. Then

$$
\begin{aligned}
& A \phi+\phi A=k \phi(k \neq 0: \text { constant }) \text { holds on } M \text { if and only if } M \text { is of type } A_{0}, A_{1} \\
& \quad \text { or } B .
\end{aligned}
$$

Proof. From (3.4) we have that $A \xi=\alpha \xi$, that is, $\xi$ is the principal curvature vector. If $A X=\lambda X$ for any $X$ in $\xi^{\perp}$, then $A \phi X=(k-\lambda) \phi X$.

By Lemma 2. $1 \alpha$ is constant. Thus we can consider the following two cases : $\alpha^{2}-4 \neq 0$ and $\alpha^{2}-4=0$.

For $\alpha^{2}-4 \neq 0$ we then have $2 \lambda-\alpha \neq 0$ by (3.2). Thus also from (3.2) it follows that $k-\lambda=(\alpha \lambda-2) /(2 \lambda-\alpha)$. Hence it follows that $2 \lambda^{2}-2 k \lambda+\alpha k-2=0$. Since $\lambda$ satisfies the above quadratic equation with constant coefficients, all principal curvatures are constant on $M$. Thus due to Theorem A, $M$ is of type $A_{1}, A_{2}$ or $B$. Suppose that $M$ is of type $A_{2}$. By Lemma 3, $1 A \phi=\phi A$ holds on $M$. This fact and (3.4) imply $2 A \phi=k \phi$. Thus from the almost contact structure it follows that $A=a I+b \eta \otimes \xi$, that is, $M$ is totally $\eta$-umblical. Then it is seen by Montiel and Romero [9] that $M$ is of type $A_{0}$ or $A_{1}$, a contradicts. Thus the type of $A_{2}$ can not occur.

Now we consider for the case $\alpha^{2}-4=0$. Let $M_{0}=\left\{x \in M \mid(2 \lambda-\alpha)_{x} \neq 0\right\}$. Then $\lambda$ also satisfies $2 \lambda^{2}-2 k \lambda+\alpha k-2=0$. Thus $\lambda$ is constant on $M_{0}$. On the other hand, we have $2 \lambda-\alpha=0$ on $M-M_{0}$. Then (3.2) gives $\alpha \lambda=2$. Thus $\lambda=$ \pm 1 on $M-M_{0}$.

The continuity of principal curvatures implies that if the set $M-M_{0}$ is not empty, then $\lambda= \pm 1$ on $M$. Hence $M$ is of type $A_{0}$.

For the case where $M_{0}$ coincides with the whole $M$, it is of type $A_{1}, A_{2}$ or $B$ and therefore it must be of type $A_{1}$ or $B$ by the same argument as that of the above half, a contradiction.

Conversely, suppose that $M$ is of type $A_{0}, A_{1}$ or $B$. It is seen by Montiel 
and Romero [9] that the type of $A_{0}$ and $A_{1}$ are the only totally $\eta$-umblical real hypersurfaces of $\boldsymbol{C} \boldsymbol{H}^{n}$. Thus it naturally satisfies $A \phi+\phi A=k \phi$.

For the type of $B$ we can take an orthonormal basis $\left\{X_{1}, \cdots, X_{n-1}, \phi X_{1}, \cdots\right.$, $\left.\phi X_{n-1}, \xi\right\}$ of $T_{x}(M)$ such that $A X_{i}=\operatorname{coth} \theta X_{i}, A \phi X_{i}=\tanh \theta \phi X_{i}, i=1, \cdots, n-1$, and $A \xi=2 \tanh 2 \theta \xi$. Then we have $A \phi X+\phi A X=(\tanh \theta+\operatorname{coth} \theta) \phi X$ for any $X$ in $T_{x}(M)$. Thus we complete the above lemma.

LEMMA 3.3. Let $M$ be a real hypersurface of $M_{n}(c), c \neq 0$. If the structure vector field $\xi$ is principal and if the second fundamental form $A$ satisfies the following quadratic formula:

$$
A^{2}+a A+c I=0\left(a^{2}-4 b \neq 0, a, b: \text { constant }\right) \text { on } \xi^{\perp},
$$

then the second fundamental form $A$ is $\eta$-parallel.

Proof. By taking covariant derivative of (3.5), we get

$$
g\left(\left(\nabla_{X} A\right) A Y, Z\right)+g\left(A\left(\nabla_{X} A\right) Y, Z\right)+\operatorname{ag}\left(\left(\nabla_{X} A\right) Y, Z\right)=0
$$

for any $X, Y$, and $Z$ in $\xi^{\perp}$.

Taking the skew-symmetric part of (3.6) and using the equation of Codazzi, we have

$$
g\left(\left(\nabla_{X} A\right) A Y, Z\right)=g\left(\left(\nabla_{Y} A\right) A X, Z\right),
$$

from which together with $g\left(A X,\left(\nabla_{Z} A\right) Y\right)=g\left(\left(\nabla_{Z} A\right) A X, Y\right)=g\left(\left(\nabla_{X} A\right) A Z, Y\right)$, we get

$$
g\left(\left(\nabla_{X} A\right) A Y, Z\right)=g\left(A\left(\nabla_{X} A\right) Y, Z\right)
$$

for any $X, Y$, and $Z$ in $\xi^{\perp}$, where we have used the fact that $\xi^{\perp}$ is invariant under the transformation of $A$ because $\xi$ is the principal curvature vector.

Combining (3.6) and (3.7), we obtain for any $X, Y$, and $Z$ in $\xi^{\perp}$

$$
2 g\left(A\left(\nabla_{X} A\right) Y, Z\right)+a g\left(\left(\nabla_{X} A\right) Y, Z\right)=0 .
$$

Transforming (3.8) with $A$ and using (3.5) again, we get

$$
2 b g\left(\left(\nabla_{X} A\right) Y, Z\right)=-\operatorname{ag}\left(A\left(\nabla_{X} A\right) Y, Z\right) .
$$

From which, substituting into (3.8), we have

$$
g\left(A\left(\nabla_{X} A\right) Y, Z\right)=0,
$$

where we have used the fact $a^{2}-4 b \neq 0$. Thus (3.9) gives $g\left(\left(\nabla_{X} A\right) Y, Z\right)=0$ for $b \neq 0$.

For the case where $b=0, a^{2}-4 b \neq 0$ implies $a \neq 0$. From which together 
with (3.8) and (3.10) it follows that $g\left(\left(\nabla_{X} A\right) Y, Z\right)=0$ for any $X, Y$ and $Z$ in $\xi^{\perp}$. Hence we get the above lemma.

These Lemmas $3.1,3.2$ and 3.3 and Theorem $A$ enable us to prove the following.

THEOREM 3.4. Let $M$ be a real hypersurface of $\mathrm{CH}^{n}$. Then the second fundamental form of $M$ is $\eta$-parallel and the structure vector field $\xi$ is principal if and only if $M$ is locally congruent to one of type $A_{0}, A_{1}, A_{2}$ or $B$.

ProOF. First we shall show that the second fundamental form of type $A_{0}$, $A_{1}, A_{2}$ or $B$ is $\eta$-parallel.

Now let $M$ be a of type $A_{0}, A_{1}$ or $A_{2}$. Then by Lemma 3.1 $A \phi=\phi A$ holds on $M$. Thus $\phi A \xi=0$ implies that $\xi$ is principal, that is, $A \xi=\alpha \xi$. From which and (3.1) it follows that

$$
A^{2}-\alpha A+I=0 \quad \text { on } \xi^{\perp} \text {. }
$$

Thus Lemma 3,3 gives that the second fundamental form is $\eta$-parallel for the case $\alpha^{2}-4 \neq 0$. For the case where $\alpha^{2}=4$ all the principal curvatures $\lambda$ are \pm 1 . Thus $M$ is of type $A_{0}$ and totally $\eta$-umblical. Hence the second fundamental form is also $\eta$-parallel in this case.

Now we consider that $M$ is of type $B$. Then by Lemma $3.2 A \phi+\phi A=k \phi$ $(k \neq 0$ : constant) holds on $M$. From which we also get $A \xi=\alpha \xi$. Thus from (3.1) it follows that

$$
A^{2}-k A-(1-\alpha k / 2) I=0 \quad \text { on } \xi^{\perp} \text {. }
$$

On the other hand, due to Berndt's classification [1] all the principal curvatures of type $B$ are given as follows: $\lambda=\operatorname{coth} \theta, \mu=\tanh \theta, \alpha=2 \tanh 2 \theta$. Since $\lambda+\mu=2 \operatorname{coth} 2 \theta=4 / \alpha, A \phi+\phi A=k \phi$ implies $k=4 / \alpha$. Hence we conclude that $k^{2}+4(1-\alpha k / 2) \neq 0$. Hence by Lemma 3.3 we also get our result.

Conversely, it suffices to show that all the principal curvatures are constant on $M$. If $A X=\lambda X$ for any $X$ in $\xi^{\perp}$, then $g\left(\left(\nabla_{Y} A\right) X, X\right)=(Y \lambda) g(X, X)$. Thus from the assumption we have that $Y \lambda=0$ for any $Y$ in $\xi^{\perp}$.

On the other hand, using equation of Codazzi and making use of (2.1) and Lemma 2.1, we get the following.

$$
\xi \lambda=g\left(\left(\nabla_{\xi} A\right) X, X\right)=g\left(\left(\nabla_{X} A\right) \xi, X\right)=0 .
$$

From these facts and Theorem $\mathrm{A}$, we conclude that $M$ is of type $A_{0}, A_{1}, A_{2}$, and $B$. This completes the above Theorem. 
REMARK. Kimura and Maeda [6] showed that a real hypersurface of $\boldsymbol{C P}^{n}$ with $\eta$-parallel second fundamental form and principal structure vector field $\xi$ is locally congruent to one of homogeneous real hypersurfaces of type $A_{1}, A_{2}$ and $B$.

\section{§4. Real hypersurfaces of $M_{n}(c), c \neq 0$, with $\eta$-parallel Ricci-tensor.}

Let $M$ be a real hypersurface of $M_{n}(c)$ with $\eta$-parallel Ricci-tensor, that is, $g\left(\left(\nabla_{X} S\right) Y, Z\right)=0$ for any $X, Y$ and $Z$ in $\xi^{\perp}$. It is easily seen that if $\xi$ is principal, then the second fundamental form $A$ of $M$ in $M_{n}(c)$ is $\eta$-parallel implies that the Ricci-tensor $S$ is $\eta$-parallel. In this section we are investigated to study this converse problem by using Kimura and Maeda's [6] result and Theorem 3.4. Then we can state another characterization as the following.

THEOREM 4.1. Let $M$ be a real hypersurface of $C P^{n}$. Then the Ricci-tensor is $\eta$-parallel and the structure vector field $\xi$ is principal if and only if $M$ is of type $A_{1}, A_{2}$ and $B$.

Proof. For any $X, Y$ in $\xi^{\perp}$, the fact that the Ricci-tensor is $\eta$-parallel implies

$$
\left(\nabla_{X} S\right) Y=-3\left(\nabla_{X} \eta\right)(Y) \xi+(X h) A Y+h\left(\nabla_{X} A\right) Y-\left(\nabla_{X} A^{2}\right) Y
$$

belongs to $[\xi]$, where $[\xi]$ means 1 -dimensional vector space spanned by $\xi$. Thus $g\left(\left(\nabla_{x} S\right) Y, Y\right)=0$ for any $Y$ in $\xi^{\perp}$. Hence if we put $A Y=\lambda Y$, then

$$
\lambda(X h)+h(X \lambda)-\left(X \lambda^{2}\right)=0 \text { for any } X \text { in } \xi^{\perp} \text {. }
$$

Also for any $Y$ in $\xi^{\perp}$ such that $A Y=\lambda Y$ we have $\left(\nabla_{\xi} A\right) Y=(\xi \lambda) Y+(\lambda I-A) \nabla_{\xi} Y$. Thus $\xi \lambda=g\left(\left(\nabla_{\xi} A\right) Y, Y\right)=g\left(\left(\nabla_{Y} A\right) \xi, Y\right)=0$. Hence the mean curvature $h$ is also constant on $\xi$-direction. Together with this fact and (4.1), we conclude that $\lambda h-\lambda^{2}$ is constant on $M$. Thus we can put as the following.

$$
\text { (4.2) } \lambda h-\lambda^{2}=a \text {, }
$$

$$
\mu h-\mu^{2}=b \text {. }
$$

By Lemma 2.2, (4.2) and (4.3) can be rewritten as follows

$$
\begin{gathered}
\lambda^{2}-h \lambda+a=0, \\
\left(2 h \alpha-\alpha^{2}-4 b\right) \lambda^{2}-\left\{\left(\alpha^{2}-4\right) h+4 \alpha-4 b \alpha\right\} \lambda-\left(2 \alpha h+b \alpha^{2}+4\right)=0 .
\end{gathered}
$$

Substituting $h \lambda=\lambda^{2}+a$ into (4.5), we then have

$$
2 \alpha \lambda^{4}-\left(2 \alpha^{2}+4 b-4\right) \lambda^{3}+2(a \alpha+2 b \alpha-3 \alpha) \lambda^{2}-\left(a \alpha^{2}-4 a+b \alpha^{2}+4\right) \lambda-2 a \alpha=0 .
$$

From which we see that $\lambda$ satisfies an algebraic equation with constant coeffici- 
ents. Thus $M$ has at most five constant principal curvatures. According to Kimura's theorem [4], $M$ is homogeneous.

On the other hand, due to Takagi's classification of homogeneous real hypersurface of $\boldsymbol{C} \boldsymbol{P}^{n}$, we conclude that $M$ is of type $A_{1}, A_{2}, B, C, D$ and $E$.

In order to prove this theorem we shall show that the shape operator is $\eta$ parallel.

Let $A(\lambda)$ be an eigenspace of $A$ with eigenvalue $\lambda$. Then the subspace $\xi_{x}^{\perp}$ of the tangent space $T_{x}(M)$ at $x$ can be decomposed as $\xi_{X}^{1}=A\left(\lambda_{1}\right) \oplus A\left(\lambda_{2}\right) \oplus \cdots$ $\oplus A\left(\lambda_{s}\right)$. Now in what follows we consider the following eigenvector such that $X \in A(\lambda), Y \in A(\mu)$ and $Z \in A(\sigma)$, where $\lambda, \mu$ and $\sigma$ are corresponding constant principal curvatures. Then we have that

$$
g\left(\left(\nabla_{X} A\right) Y, Z\right)=(\mu-\sigma) g\left(\nabla_{X} Y, Z\right) .
$$

On the other hand, from the $\eta$-parallel Ricci-tensor it follows that

$$
(h-\mu-\sigma) g\left(\left(\nabla_{X} A\right) Y, Z\right)=0 .
$$

For the case where $\mu=\sigma$, (4.7) implies that $g\left(\left(\nabla_{X} A\right) Y, Z\right)=0$. Thus it suffices to show that the shape operator is $\eta$-parallel for the case where $\mu \neq \sigma$.

In the case where $h-\mu-\sigma \neq 0,(4.8)$ gives our result. Thus it remains to consider for the case where $h-\mu-\sigma=0$. Thus the $\eta$-parallel Ricci-tensor gives

$$
g\left(\left(\nabla_{Y} S\right) X, Z\right)=(h-\lambda-\sigma) g\left(\left(\nabla_{Y} A\right) X, Z\right)=0 .
$$

If $\lambda \neq \mu$, then $h-\mu-\sigma=0$ implies $h-\lambda-\sigma \neq 0$. From which together with (4.9) it follows $g\left(\left(\nabla_{X} A\right) Y, Z\right)=g\left(\left(\nabla_{Y} A\right) X, Z\right)=0$. If $\lambda=\mu,(4.7)$ gives $g\left(\left(\nabla_{X} A\right) Y, Z\right)=$ $g\left(\left(\nabla_{Z} A\right) X, Y\right)=0$. Summing up, we conclude that the shape operator is $\eta$ parallel. Thus, due to Kimura and Maeda's Theorem [6], $M$ is of type $A_{1}, A_{2}$ and $B$.

Conversely, if $M$ is of type $A_{1}, A_{2}$ or $B$, then by Kimura and Maeda's Theorem [6] the second fundamental form is $\eta$-parallel and its structure vector field $\xi$ is principal. Since $\eta$-parallel second fundamental form with the principal structure vector $\xi$ implies $\eta$-parallel Ricci-tensor, we get the above Theorem.

REMARK. Kimura [5] showed that a real hypersurface of $\boldsymbol{C P}^{n}$ with the condition $\left(\nabla_{X} S\right) Y=c\{g(\phi A X, Y)+\eta(Y) \phi A X\}$, where $c$ is constant, is locally congruent to homogeneous hypersurfaces with 2 or 3 distinct principal curvatures. Thus this condition implies that the Ricci-tensor $S$ is $\eta$-parallel and structure vector field $\xi$ is principal. 
On the other hand, for a real hypersurface of $\boldsymbol{C H}^{n}$ we get the following.

THEOREM 4.2. Let $M$ be a real hypersurface of $\boldsymbol{C H}^{n}, n \geqq 2$. Then the Rcci-tensor is $\eta$-parallel and the structure vector field $\xi$ is principal if and only if $M$ is of type $A_{0}, A_{1}, A_{2}$ or $B$.

Proof. The converse is trivial by Theorem 3.4.

Let $M$ be a real hypersurface of $\boldsymbol{C H}^{n}$ with $\eta$-parallel Ricci-tensor and principal structure vector field $\xi$. Then similarly as in Theorem 4.1 we can put

$$
\begin{aligned}
& \lambda h-\lambda^{2}=a, \\
& \mu h-\mu^{2}=b .
\end{aligned}
$$

By Lemma 2.1 we can consider the following two cases.

CASE I. $\quad \alpha^{2}-4 \neq 0$.

Then $2 \lambda-\alpha \neq 0$. In fact, suppose that $2 \lambda-\alpha=0$. Then (3.2) gives $\alpha \lambda=2$. Together with this fact we have $\alpha^{2}-4=0$, a contradiction. Thus from (3.2) it follows $A \phi X=\mu \phi X, \mu=(\alpha \lambda-2) /(2 \lambda-\alpha)$. From which, substituting (4.14), then we get

$$
\left(2 \alpha h-\alpha^{2}-4 b\right) \lambda^{2}+\left\{4 \alpha+4 b \alpha-\left(\alpha^{2}+4\right) h\right\} \lambda+\left(2 \alpha h-4-b \alpha^{2}\right)=0 .
$$

Substituting (4.13) into (4.15), then $\lambda$ satisfies the following equation with constant coefficients

$$
2 \alpha \lambda^{4}-2\left(\alpha^{2}+2 b+2\right) \lambda^{3}+2 \alpha(a+2 b+3) \lambda^{2}-\left(a \alpha^{2}+b \alpha^{2}+4 a+4\right) \lambda+2 a \alpha=0 .
$$

In the case where $\alpha=0, a=-1$ and $b=-1$, coefficients of the above equation are all vanishing. Thus it suffices to prove that principal curvatures are also constant on $M$ in this case.

For the case where $a=-1$, and $b=-1$ it follows from (4.13) and (4.14) that $\lambda=\mu$ or $h=\lambda+\mu$. Since $\mu=-1 / \lambda$ for $\alpha=0, \lambda=\mu$ implies $\lambda^{2}+1=0$. This contradicts. Thus we have $h=\lambda+\mu$. From which together with $h=m_{1} \lambda+m_{2}(-1 / \lambda)$ for $\alpha=0$, it follows that $\left(m_{1}-1\right) \lambda^{2}-\left(m_{2}-1\right)=0$. Since $m_{1} \neq 1$, principal curvatures are constant on $M$ in this case. Hence all principal curvatures are constant on $M$. Thus due to Theorem A we conclude that $M$ is of type $A_{1}, A_{2}$ or $B$.

CASE II. $\alpha^{2}=4$.

Now we consider for the case $\alpha=2$. Then (3.2) gives 


$$
(\lambda-1) A \phi X=(\lambda-1) \phi X .
$$

Let us take an open set $M_{0}=\{x \in M \mid \lambda \neq 1\}$. Then $A \phi X=\phi X$. Thus $\mu=1$. From which and (4.14) it follows $h=b+1$ on $M_{0}$. Since $\lambda=1$ on $M-M_{0}$, also from (4.13) it follows $h=a+1$. Hence $h$ is constant and $a=b$ on $M$. Thus $\lambda$ satisfies a quadratic equation with constant coefficients : $\lambda^{2}-h \lambda+a=0$. Hence all principal curvatures are constant on $M$.

Similarly, for the case $\alpha=-2$ we also get the same conclusion. By virtue of Theorem A, $M$ is of type $A_{0}, A_{1}, A_{2}$ or $B$. Since $\alpha= \pm 2$, then $M$ is of type $A_{0}$.

\title{
References
}

[1] Berndt, J., Real hypersurfaces with constant principal curvatures in complex hyperbolic space, J. reine angew, Math. 395 (1989), 132-141.

[2] Cecil T.E. and Ryan., P. J., Focal sets and real hypersurfaces in complex projective space, Trans. Amer. Math. Soc. 269 (1982), 481-499.

[3] Ki, U.H. and Suh., Y.J., On real hypersurfaces of a complex space form, to appear in Okayama Math. J.

[4] Kimura, M., Real hypersurfaces and complex submanifolds in a complex projective space, Trans. Amer. Math. Soc. 296 (1986), 137-149.

[5] Kimura, M., Real hypersurfaces of a complex projective space, Bull. Austral. Math. Soc. Vol. 33 (1986), 383-387.

[6] Kimura, M. and Maeda, S., On real hypersurfaces of a complex projective space, Math. Z. 202 (1989), 299-311.

[7] Maeda, Y., On real hypersurfaces of a complex projective space, J. Math. Soc. Japan 28 (1976), 529-540.

[8] Montiel, S., Real hypersurfaces of a complex hyperbolic spaces, J. Math. Soc. Japan 37 (1985), 515-435.

[9] Montiel, S. and Romero, A., On some real hypersurfaces of a complex hyperbolic space, Geometriae Dedicata, 20 (1986), 245-261.

[10] Takagi, R., On homogeneous real hypersurfaces of a complex projective space, Osaka J. Math. 10 (1973), 495-506.

[11] Takagi, R., Real hypersurfaces in a complex projective space with constant principal curvatures. I, II. J. Math. Soc. Japan 27 (1975), 43-53, 507-516.

[12] Yano, K. and Kon, M., CR-submanifolds of Kaehlerian and Sasakian manifold, Birkhauser 1983.

\author{
University of Tsukuba \\ Institute of Mathematics \\ Ibaraki, 305 \\ Japan \\ and \\ Andong University \\ Department of Mathematics \\ Andong, 760-749 \\ Korea
}

